\title{
Autogenous and Reactive Obsessions in Obsessive-Compulsive Disorder: A Keyness and Collocation Study \\ Stella Kim ${ }^{1}$ and Cass Dykeman ${ }^{1}$ \\ ${ }^{1}$ Oregon State University
}

This preprint has been submitted to a peer-review journal. Correspondence concerning this manuscript should be addressed to Stella Kim at kimstel@oregonstate.edu

\begin{abstract}
This study explored the subjective experiences of individuals with autogenous and reactive forms of obsessions in obsessive-compulsive disorder. Past studies have found greater levels of shame, stigma, and concealment for those experiencing lesser-known forms of OCD, particularly those that deal with sexual themes. This study looked at what words most differentiated (i.e, keyness analysis) these subtypes. In addition, the word networks (i.e., collocates) of the words that most differentiated the subtypes were examined. The keywords "sexual" and "POCD" had the strongest keyness in the autogenous corpus. Contamination and checking had the strongest keyness in the reactive corpus. The collocates of the node words in the autogenous corpus show a focus on the intrusive thoughts themselves, whereas the collocates in the reactive corpus focus on performing compulsions such as checking. Limitations, clinical implications, and future research recommendations are discussed.

Keywords: reactive OCD, autogenous OCD, obsessive compulsive disorder, corpus linguistics

\section{Introduction}

For different people, what comes to mind when they think of obsessive compulsive disorder can relate to fear of germs, excessive washing of hands, compulsive need to put things in order, or habit of counting things. However, people who suffer from OCD also experience thoughts such as violent and sexual obsessions. Many of these individuals fear that the general population, including mental health clinicians, will not be able to understand their symptoms and may even end up facing criminal consequences due to the nature of their intrusive thoughts. With the increased accessibility of technology and online forums, people who are unable to access treatment for lesser-known types of OCD can express themselves anonymously and crowdsource support and information.
\end{abstract}


Written narratives can provide a wealth of information about psychological processes through examining linguistic and psychological dimensions of online posts. The goal of such an examination would be to illuminate the experiences of the different types of obsessions.

This study sought to contribute research to the existing literature about the linguistic processes of individuals who live with OCD. To date, there have been only two corpus linguistics studies that looked at OCD, and they looked only at the sexual orientation subtype of OCD (Coimbra-Gomes, 2020; CoimbraGomes \& Motschenbacher, 2019). This study also aims to enrich the knowledge base for clinicians to understand the psychological and linguistic processes associated with different subtypes of OCD. Linguistic processes can uncover connections in language and discourse (Brezina, 2018). The lack of access to trained clinicians is a major issue in treating individuals with OCD; research has found that clinicians need to be more educated to learn about the heterogeneity of OCD (Glazier et al., 2013). Clinician incompetence continues to be a major issue, as traditional cognitive-behavioral therapy (CBT) does not adequately treat symptoms of OCD, and the gold standard for OCD, exposure and response prevention (ERP), is not commonly taught in training programs. This study can help clinicians learn about subtypes of OCD while also understanding the unique psychological processes associated with each subtype. In particular, feelings of guilt and shame have been found to be associated more with the autogenous subtype of OCD (Lee \& Kwon, 2003). Targeting specific psychological processes unique to the subtypes can help to build trust between clients and clinicians, as well as to choose interventions that target specific emotional distress linked to that subtype.

In reviewing the literature on language and $\mathrm{OCD}$, four themes emerged: (a) features of autogenous obsessions, (b) features of reactive obsessions, (c) avoidance of formal treatment due to stigma, and (d) common psychological themes of help-seeking individuals with OCD on online forums. After a review of the literature, the research questions for this study will be discussed.

Autogenous obsessions tend to occur without external stimuli to trigger the intrusive thought and contain ego-dystonic themes (Lee \& Kwon, 2003). These types of intrusive thoughts are considered by individuals experiencing them to be repugnant, taboo, and ego-dystonic, and therefore are experienced as threatening to their self-perceptions (Moulding et al., 2013). In particular, autogenous obsessions about sexual themes such as pedophilia are more likely to be perceived by these individuals as less acceptable than other types of obsessions (Cathey \& Wetterneck, 2013). Autogenous obsessions are therefore less likely to 
be disclosed to others due to fears of social rejection and disapproval. Another feature of autogenous obsessions is the construct of mental contamination, which individuals can experience after having a repugnant thought (Rachman, 2006). Scrupulosity or religious OCD has been found to be associated with a construct in which individuals feel an inner sense of dirtiness without the presence of an external trigger (Fergus \& Rowatt, 2014). Autogenous obsessions, when compared to reactive obsessions, tend to cause intense emotional distress in individuals due to the ego-dystonic nature of the thoughts as well as the negative inferences about the self that are created from them.

Reactive obsessions are triggered by external stimuli and set off a set of compulsive behaviors to undo the threat of these thoughts. These types of obsessions are more often described as being realistic due to having an identifiable trigger that the individual experiences (Lee \& Kwon, 2003). These obsessions are related to contamination, symmetry, mistakes, and accidents. In contrast to autogenous obsessions, reactive obsessions provoke anxiety not so much due to the thoughts themselves but more because of their potential negative consequences (Lee et al., 2005). Reactive obsessions also tend to have more observable compulsions such as washing, checking, and ordering, due to the need to prevent bad things from occurring (Moulding et al., 2006). Individuals experiencing autogenous and reactive subtypes of OCD often express hesitation to seek treatment due to a myriad of fears and social stigmas.

Individuals with OCD anticipate stigma from others and experience selfstigma. They fear that they will be viewed as mentally "ill" or not "normal" by others. They also experience a crisis of self-conception as many of their obsessions are intrusive and go against their beliefs. In particular, repugnant or forbidden obsessions related to sexual themes and harm can carry more stigma than other types of obsessions. A study by Cathey and Wetterneck (2013) found that sexual obsessions were met with more social rejection than contamination obsessions. Within sexual obsessions, individuals with pedophilia OCD (POCD) experience much more stigma than those with sexual orientation OCD (SO-OCD; Snethen \& Warman, 2018). Similarly, Homonoff and Sciutto (2019) found that individuals with harm OCD were met with more stigmatizing attitudes by others than were individuals with "just right" obsessions. These studies highlight the challenges that individuals with autogenous obsessions experience in sharing about their symptoms with others as well as having to reckon with ego-dystonic thoughts within themselves.

In addition to stigma from others and self-stigma, a few studies have looked at clinicians' stigmatization of attitudes toward individuals with various 
types of obsessions. Specifically, clinicians were found to hold the most socially rejecting attitudes toward individuals who experienced sexual obsessions.

Interestingly, researchers also found that contamination was met with as much stigma as harm obsessions. Scrupulous or religious obsessions were met with the least amount of social rejection (Steinberg \& Wetterneck, 2017). Psychiatrists also reported negative attitudes toward their patients with OCD, expressing annoyance that they talked too much, were too needy, were not compliant with treatment, and were difficult to treat (Kusalaruk et al., 2015). In addition to stigma, many clinicians failed in their differential diagnosis to correctly identify taboo thoughts and autogenous obsessions as OCD symptoms. They were the least accurate in identifying sexual obsessions about sexual orientation (77.0\%), sexual obsessions about pedophilia (42.9\%), aggressive obsessions (31.5\%), and religious obsessions (28.8\%; Glazier et al., 2013). Many individuals who fear stigma and the consequences of misidentification of OCD turn to online forums to seek support and information about their symptoms instead of seeking formal treatment.

Due to stigma and shame, individuals experiencing symptoms of OCD experience fear of seeking formal treatment. Those experiencing repugnant or forbidden obsessions related to the autogenous subtype of OCD were more likely to express a fear of involuntary hospitalization than those with other types of obsessions due to being misdiagnosed by clinicians (Glazier et al., 2015). A qualitative study found that individuals did not seek formal treatment due to external factors such as fearing clinicians' reactions to their symptoms and a fear of criminalization, particularly among those with sexual obsessions. Glazier et al. (2015) also found internal psychological barriers such as individuals thinking that their symptoms were not severe enough compared with other psychological disorders to seek help or that they did not deserve to get help (Robinson et al., 2017). They were also found to have higher levels of self-concealment compared to individuals without OCD, and this self-concealment was more prevalent in individuals experiencing repugnant obsessions (Wheaton et al., 2016). Due to these internal and external barriers, formal treatment may not be a plausible option for many individuals suffering with OCD. With the increased use of technology and communication, other methods such as virtual communities have become options for obtaining support.

Individuals with OCD seek help on online forums for various reasons. Among cited reasons for using online communication are the amount of easily accessible information about symptoms and treatment, tips about living with OCD, and being able to communicate with a supportive community (Stein, 1997). Virtual space for blogs and forum posts written by individuals with OCD 
can provide insight into clinical implications as well. A study by Campbell and Longhurst (2013) found that there were gendered differences in the ways the posters expressed their experiences with OCD. Females were more likely to conceptualize it as a journey, while males saw it as a battle. This could be helpful in conceptualizing how an individual may approach treatment and how adaptable they might be to challenges they face. They also found that individuals were more likely to post when in distress to gain social support from others (Campbell \& Longhurst, 2013). These few studies show that a closer examination of online communication can uncover individuals' subjective experiences and enrich the understanding of individuals' experiences and how to treat nuances of OCD.

There were six research questions that guided this study. These questions were:

RQ1: What words most differentiate autogenous obsession posts from reactive obsessions posts?

RQ2: What words most differentiate reactive obsession posts from autogenous obsession posts?

RQ3: In online posts of individuals experiencing autogenous obsessions, what are the collocates of the word with the strongest keyness?

RQ4: In online posts of individuals experiencing autogenous obsessions, what are the collocates of the word with the second strongest keyness?

RQ5: In online posts of individuals experiencing reactive obsessions, what are the collocates of the word with the strongest keyness?

RQ6: In online posts of individuals experiencing reactive obsessions, what are the collocates of the word with the second strongest keyness?

\section{Method}

\section{Design}

A synchronic corpus linguistic design was used for this study. The variables were word usage uniqueness (i.e., keyness) and word relatedness (i.e., collocation). The corpora were drawn from online posts by individuals with autogenous obsessions and reactive obsessions. The level of measure was continuous. The unit of analysis was individual words.

To determine the power required for this study, a power analysis was done in $\mathrm{G}^{*}$ Power 3.1 to determine the requisite sample size for an adequately powered study (Faul et al., 2009). Since the planned analyses involved a chisquare derivative, Cohen's $w$ was the relevant effect size. The Cohen's $w$ was 
drawn from an anxiety study (Helle et al., 2016). The following input parameters were employed: (a) test family $=\chi^{2}$ tests; (b) statistical test $=$ goodness-of-fit tests: contingency tables; (c) power analysis $=$ compute required sample size, given $\alpha$, power, and effect size; (d) effect size $w=0.17$; (e) $\alpha$ error probability $=.01$, (f) power $(1-\beta$ error probability $)=0.80$; and $(\mathrm{g})$ degrees of freedom $=1$. The $\mathrm{G}^{*}$ Power 3.1 output parameters were: (a) total sample size $=405$ and (b) actual power $=0.80$.

\section{Corpus}

The corpus for this study was the same as used by Kim and Dykeman (2020) for a study of OCD subtypes examining personological and function word use. The register was blogging, and the subregister was blogging about OCD. There were two subcorpuses: autogenous and reactive.

\section{Reactive Subcorpus}

Register, Scope, and Sources. The scope was public posts on OCD made by individuals in Reddit API. This corpus contained posts written by individuals with the reactive type of obsessions of OCD identified by the cognitive processes model of Kwon and Lee (2003). The autogenous subtype corpus included posts about symmetry, ordering, checking, and contamination. Reddit was scraped on January 25, 2021, and resulted in 949,264 tokens in the autogenous corpus.

Preprocessing. All words were converted to lowercase letters, punctuation was removed, and all words were converted to a .txt file. Words that were not single-token, such as HOCD (harm obsessive compulsive disorder), were kept as written in the forums. Stopwords were filtered out to focus on meaningful words. The stopword list used was from the_NLTK (Bleier, 2021). The corpora were checked for non-standard orthography including typing errors and spelling errors. All abbreviations were also changed to spell out the full words (e.g., "idk" to "I don't know"). The corpora were converted to .txt files and uploaded into \#Lancsbox.

\section{Autogenous Subcorpus}

Register, Scope and Sources. The register was the same as for the reactive corpus. The scope was posts that were classified as autogenous obsessions. The autogenous subtype corpus includes repugnant obsessions such as 
harm OCD, scrupulosity OCD, pedophilia OCD, and responsibility OCD that appeared from January 25, 2021, to the oldest existing post on Reddit. The total number of tokens for the reactive corpus was 1,413,950.

Preprocessing. The preprocessing was the same as for the reactive corpus.

\section{Measures}

\section{Node Words}

A node word is the focus of analyses in a collocation study. A node word is the word of interest or "any word which the user wishes to interrogate" (Baker, 2016, p. 141). Node words are used to rank collocates on how frequently they co-occur in the corpus. Collocation studies are used to study the strength of the relationship between a node and collocate (Brezina et al., 2015).

\section{Keyness}

Keyness describes words that are important in a corpus and that indicate what a text is really about (Scott \& Tribble, 2006). These words occur frequently in the specific corpus that are infrequent in others. Keyness analysis identifies words that are salient and reflective of the subject of the text (Jensen, 2020).

\section{Collocation}

Collocation is the repeated co-occurrence of words in texts. Collocations create opportunities to study the connection between words, and corpus techniques uncover the strength of associations between words that frequently co-occur (Brezina, 2018).

\section{Apparatus}

\section{WordSmith Tools 8.0}

WordSmith Tools is a program that examines how words are used in texts. Keywords were identified by comparing the tokens of the corpora to determine the frequency in which they appear.

\section{Collocation Graph (GraphColl)}

GraphColl is an analysis tool in \#Lancsbox that analyzes collocation networks (Brezina et al., 2018). After a corpus is loaded, node words are searched in the corpus to identify collocates using specific parameters for the search settings. This tool visually displays linguistic collocations of identified node 
words. Node words and their collocates are represented with lines that represent the strength of their relationships. The length of the line is inversely proportional to the strength of the relationship between the words. Darker colors represent higher frequencies of collocates, and the position of the collocates around the node words represent the position (to the left or right of node) where they tend to appear in the corpora (Brezina et al., 2015).

\section{Data Analysis}

For the first two research questions, a log-likelihood ratio test $\left(G^{2}\right)$ was used for analysis of keywords in the autogenous and reactive subcorpora using procedures from Egbert and Biber (2019). The top ten keywords for each corpus are reported. The effect size was Bayesian information criterion (BIC). The BIC strength descriptors were drawn from (Fabozzi et al., 2014). Wordsmith Tools 8.0 (Smith, 2020) was used for the keyness analysis with the alpha set at 01.

In terms of RQs 3-6, the collocation parameters were: statistic ID $=5$, statistic name $=$ Mutual Information $\left(\mathrm{MI}^{3}\right)$, the span of the left and right context $=5$, statistic cut-off value $=20.0$, minimum collocate frequencies in the corpus $=20$, and filter $=$ NLTK stopwords (Brezina, 2018). All analyses occured via \#Lancsbox's feature (Brezina et al., 2018).

\section{Results}

The results for RQs 1-2 were identified using Wordsmith Tools 8.0. The top ten keywords with the strongest keyness for the autogenous and reactive corpuses can be found in Table 1. RQs 3-6 looked at collocates with the strongest keyness, and the complete GraphColls are in Figures 1-4. Supplemental results including proportional data can be found on this research project's Open Science Foundation website (https://osf.io/43ame/).

\section{Discussion}

This study looked at collocations of words in online posts of individuals with autogenous and reactive obsessions. The first two research questions looked at which words in the reactive and autogenous corpuses that most differentiated each corpus from the other. RQs 3-4 looked at the collocates of the words "checking" and "sexual," which were the words with the strongest keyness in the reactive and autogenous corpuses, respectively. RQs 5-6 looked at the collocates

of "contamination" and "POCD" which were the words with the second strongest keyness. 
RQ1 looked at what words most differentiated the autogenous corpus from the reactive corpus. The tokens with the strongest keyness were "sexual" and "POCD." The most basic explanation for this finding is that amongst the heterogeneous themes of OCD, unwanted sexual or aggressive thoughts have been found to be particularly distressing and repugnant to the individual. These types of obsessions have been linked to greater levels of distress and negative self-beliefs (Moulding et al., 2014). A subset of unwanted sexual thoughts often relates to sexually harming children and fearing being a pedophile (Bruce et al., 2018). In addition to the nature of these obsessions, as well as the fear of being misdiagnosed and not receiving the proper treatment from mental health professionals could lead to increased levels of distress and hopelessness, thus prompting individuals suffering from POCD to seek advice in online forums.

RQ2 looked at the words that most differentiate the reactive corpus from the autogenous corpus. The words with the strongest keyness in this corpus were checking and contamination. One explanation of these results is that these two words are related to a very common compulsion and obsession experienced by many OCD sufferers. A more nuanced explanation could be that individuals post frequently about checking rituals on Reddit because consistent with past research, there is an inflated perception of responsibility for harm that individuals then seek relief from by performing compulsions (Foa et al., 2002). A hallmark of reactive obsessions is that they are triggered by external stimuli (Lee \& Kwon, 2001), so it would make sense that individuals would then act to neutralize the threats posed by the intrusive thoughts. Similarly, contamination fears are also associated with inflated estimates of threat and responsibility (Wheaton et al., 2010).

In terms of RQ3, two main themes emerged about the collocates for the word "sexual." The first theme has to do with the ego-dystonic nature of the obsessions and includes the collocates "thoughts," "intrusive," "assault," "abuse," and "never." It is noteworthy that the strongest collocate is "thoughts," which means that individuals posting on the Reddit forum about sexual OCD were posting about their obsessions, not their compulsions. This is consistent with the autogenous subtype of OCD where individuals experience intrusive thoughts but do not have physical, observable compulsions (Lee \& Kwon, 2001). The top words used to describe these intrusive thoughts are "assault" and "abuse," which may indicate that these individuals possess the insight to know that the thoughts they experience are not in line with their actual sexual desires and morals which would cause harm to others. This may be further supported by the words "intrusive" and "never," the latter which is often used to describe how the individual would never act on these thoughts or have the desire to do so. 
The second theme that emerged was about the narrative of what the thoughts might mean for the individual. The words "attraction" and "orientation" were strong collocates and could indicate the doubt that OCD introduces for individuals about sexual orientation. Because a clinical feature of sexual obsessions is the distress related to what the meaning of having these types of thoughts is, these collocates could be indicative of the rumination with which the individual engages (Vella-Zarb et al., 2017).

RQ4 looked at the collocates of "POCD," which had the second strongest keyness in the autogenous corpus. Similar to the previous research question, the word "thoughts" was a strong collocate. This could indicate that the individuals experiencing obsessions about pedophilia experience intrusive obsessions and write about them in the Reddit forums. Because "thoughts" is a strong collocate and is linked to the obsessions, this further supports Lee and Kwon's (2003) model of OCD which posits that autogenous obsessions do not have to be triggered by specific external stimuli. Once these thoughts are introduced, subsequent thoughts could also be related to the rumination individuals engage in to reassure themselves. Another theme related to POCD can be seen with the collocates "HOCD" and "ROCD." These are subtypes of sexual obsessions that are related to sexual orientation and relationship substantiation. In both cases, an explanation for why these were strong collocates is that the obsessions have to do with attraction to others. An individual experiencing pedophilic intrusive thoughts could very well then experience anxiety and distress about their own sexual identity or relationships, as catastrophic thoughts about being in the wrong relationship were found to predict the presence of ROCD (Melli et al., 2018). It is also possible that these three subtypes of sexual obsessions can be related, as the rituals can be similar in nature. Individuals will often look at photos, watch movies, or look at other stimuli and scan their bodies for signs of arousal that would support or discredit the obsessive thought (Doron et al., 2016; Vella-Zarb et al., 2017; Williams, 2008).

RQ5 looked at the collocates of the word with the strongest keyness in the reactive corpus. The collocates for checking included words describing the common compulsive rituals that accompany reactive obsessions. To neutralize the threat posed by the intrusive thought, words that accompanied checking such as "things," "locks," and "doors" substantiate the cognitive processing model of OCD (Lee \& Kwon, 2001) by showing how individuals modify their environments to perform observable acts. The other collocates such as "sure" and "constantly" convey one of the hallmarks of OCD, which is uncertainty. The rituals performed in response to reactive obsessions serve to ascertain some level of reassurance and 
certainty that the rituals will prevent a negative outcome for the individual. These collocates are consistent with past research which showed that IU (intolerance of uncertainty) is especially prevalent and significant in individuals with checking rituals (Tolin et al., 2003).

RQ6 addressed the collocates of the word "contamination," which had the second strongest keyness in the reactive corpus. The words "fear," "fears," and "germs" were strong collocates. There are a few explanations for this result. The first is that reactive obsessions, such as intrusive thoughts about contamination, are triggered by external stimuli in the immediate environment, such as touching a doorknob. This could then trigger the fearful thought that the individual may get sick, contract an illness, or pass the germs onto someone else. Thus, fears of unwanted and negative consequences are a hallmark of reactive obsessions. More specifically, research about contamination OCD has shown that there are specific dimensions to these fears that center around disgust responses. Higher ratings of disgust propensity and sensitivity predicted contamination obsessions, with fears of contagions underlying the feelings of disgust (Olatunji et al., 2004). Another finding is that the word "anyone" was also a strong collocate for "contamination." Compared to the autogenous corpus, this is the only keyword that includes a word that references others. Past research has shown that contamination OCD is accompanied by inflated sense of responsibility, as well as interpersonal concerns about protecting others (Ashbaugh et al., 2006). Because there is an inflated sense of responsibility to prevent harm from coming to others, there is also excessive interpersonal reassurance seeking involved with this form of OCD, which could also explain the frequency of other-directed words such as "anyone" appearing in this corpus (Leonart \& Radomsky, 2019).

This study used a collocation study design which has two main limitations. First, the corpus was selected and built from one source, which was the OCD subreddit forum. Additional sources of posts could have diversified the corpora, as Reddit is an anonymous forum that specific users might utilize to write about topics that they would not share elsewhere. The second limitation is that while collocations provide important insight into language usage and expressions of subjective experiences, it does not equate to definitive conclusions about the context in which these words appeared in the corpuses.

There are three clinical implications from these findings. The first implication is that this study supports the model of OCD developed by Lee and Kwon (2001) that shows the differentiation between autogenous and reactive obsessions and their symptom manifestations. Clinicians must be trained to understand the nuances and heterogeneity of the various subtypes of OCD to be 
able to competently assess and treat these symptoms. Secondly, the collocations for both the reactive and autogenous corpuses show that there are linkages between the underlying psychological processes, such as the connection of pedophilia OCD to ruminations about sexual orientation (HOCD) and relationship substantiation (ROCD), and the linkage between responsibility, disgust, and harm in contamination OCD. These are important nuances that need attention in treatment, in addition to the gold standard ERP, for the progress and outcomes of interventions to be successful. The third clinical implication is that clinicians must incorporate the interpersonal aspects of OCD. For example, contamination fears could affect family members, friends, and other familiar people close to the individual by involving them in the decontamination, cleaning, and disinfecting rituals. These individuals must also be involved in treatment to learn to respond effectively to reassurance seeking from the affected individual, as this is a form of avoidance that runs counter to the goals of ERP. Similarly, sexual obsessions also involve interpersonal components as the affected individual will frequently perform checking rituals to confirm or invalidate their obsessions about attraction to their partners.

In terms of research implications, the research body needs more studies on clinician competency, familiarity, and confidence in working with various presentations of OCD. This study shows the heterogeneity and complex nuances of OCD, as well as the shortcomings of clinician knowledge on treating repugnant obsessions such as POCD. The lack of clinician familiarity with autogenous obsessions could lead to OCD being mistaken for sexual paraphilias and cause more harm to the individual with OCD. It also can perpetuate the cycle of shame and secrecy that individuals experiencing repugnant obsessions experience. More studies about the interpersonal aspects of OCD would also add to the complexity and nuanced understanding of the various subtypes of obsessions. There have been a few studies that show the effects of family and partners' responses to OCD that affect symptoms, and it is clear from the results of this study that psychological processes such as harm, ruminations about responsibility to others, and worries about relationships play a large role in OCD. 


\section{References}

Ashbaugh, A. R., Gelfand, L. A., \& Radomsky, A. S. (2006). Interpersonal aspects of responsibility and obsessive compulsive symptoms. Behavioral and Cognitive Psychotherapy, 34, 151-163. https://doi.org/10.1017/S1352465805002699

Balci, V., \& Sevincok, L. (2010). Suicidal ideation in patients with obsessivecompulsive disorder. Psychiatry Research, 175, 104-108.

Belloch, A., Morillo, C., \& Garcia-Soriano, G. (2006). Subtipos de obsesiones y su relación con síntomas obsesivo-compulsivos, creencias disfuncionales y estrategias de control [Obsession subtypes: Relationships with obsessivecompulsive symptoms, dysfunctional beliefs and thought control strategies]. Revista de Psicopatología y Psicología Clínica, 11(2), 65-78. https://doi.org/10.5944/rppc.vol.11.num.2.2006.4018

Bleier, S. (2021). NLTK's list of English stopwords. https://gist.github.com/sebleier/554280\#file-nltk-s-list-of-englishstopwords

Brezina, V. (2018). Statistics in corpus linguistics: A practical guide. Cambridge University Press.

Brezina, V., McEnery, T., \& Wattam, S. (2015). Collocations in context: A new perspective on collocation networks. International Journal of Corpus Linguistics, 20(2), 139-173. https://doi.org/10.1075/ijcl.20.2.01bre

Bruce, S. L., Ching, T. H., \& Williams, M. T. (2018). Pedophilia-themed obsessive-compulsive disorder: assessment, differential diagnosis, and treatment with exposure and response prevention. Archives of Sexual Behavior, 47, 389-402. https://doi.org/10.1007/s10508-017-1031-4

Chiang, B., Purdon, C., \& Radomsky, A. (2016). Development and initial validation of the fear of guilt scale for obsessive-compulsive disorder (OCD). Journal of Obsessive-Compulsive and Related Disorders, 11, 6373. https://doi.org/10.1016/j.jocrd.2016.08.006

Coimbra-Gomes, E., \& Motschenbacher, H. (2019). Language, normativity, and sexual orientation obsessive-compulsive disorder (SO-OCD): A corpusassisted discourse analysis. Language in Society, 48(4), 565-584. https://doi.org/10.1017/S004740451900042

Doron., G., Derby, D., Szepsenwol, O., Nahaloni, E., \& Moulding, R. (2016). Relationship obsessive-compulsive disorder: Interference, symptoms, and maladaptive beliefs. Frontiers in Psychiatry, 7, Article 58.

https://doi.org/10.3389/fpsyt.2016.00058

Foa, E., Sacks, M. B., Tolin, D. F., Prezworksi, A., \& Amir, N. (2002). Inflated perception of responsibility for harm in OCD patients with and without checking compulsions: A replication and extension. Journal of Anxiety Disorders, 16(4), 443-453. https://doi.org/⒑1016/s0887-6185(02)00128-7 
Gershkovich, M., Wheaton, M. G., \& Simpson, H. B. (2017). Management of treatment-resistant obsessive-compulsive disorder. Current Treatment Options in Psychiatry, 4, 357-370. https://doi.org/10.1007/s40501-017$\underline{0127-8}$

Glazier, K., Wetterneck, C., Singh, S., \& Williams, M. (2015). Stigma and shame as barriers to treatment for obsessive-compulsive and related disorders. Depression and Anxiety, 4(3), 1000191. http://dx.doi.org/10.4191/21671044.1000191

Hartman, J. (2018). Constructions of contrast in spoken testimonials on obsessive compulsive disorder. Language and Cognition: An Interdisciplinary Journal of Language and Cognitive Science, 10(1), 83-109. https://doi.org/10.1017/langcog.2017.18

Jacoby, R. J., Leonard, R. C., Riemann, B. C., \& Abramowitz, J. S. (2016). Selfpunishment as a maladaptive thought control strategy mediates the relationship between beliefs about thoughts and repugnant obsessions. Cognitive Therapy and Research, 40, 179-187. https://doi.org/10.1007/s10608-015-9741-1

Jensen, K. E. (2020). Corpus-methodology and discursive conceptualizations of depression. In M. Filimowicz, \& V. Tzankova (Eds.), Reimagining communication: Meaning (pp. 64-82). Routledge.

Keleş Altun, İ., Uysal, E., \& Özkorumak Karagüzel, E. (2017). Differences between autogenous and reactive obsessions in terms of metacognitions and automatic thoughts. Neuropsychiatric Disease and Treatment, 13, 2977-2985. https://doi.org/10.2147/NDT.S15108

Keyes, C., Nolte, L., \& Williams, T. I. (2018). The battle of living with obsessive compulsive disorder: A qualitative study of young people's experiences. Child and Adolescent Mental Health, 23(3), 177-184. https://doi.org/10.1111/camh.12216

Knapton, O. (2016). Experiences of obsessive-compulsive disorder: activity, state, and object episodes. Qualitative Health Research, 26(14), 2009-2023. https://doi.org/10.1177/1049732315601666

Lee, H. J., \& Kwon, S. M. (2003). Two different types of obsession: Autogenous obsessions and reactive obsessions. Behaviour Research and Therapy, 41(1), 11-29. https://doi.org/10.1016/s0005-7967(01)00101-2

Lee, H. J., Lee, S. H., Kim, H. S., Kwon, S. M., \& Telch, M. J. (2005). A comparison of autogenous/reactive obsessions and worry in a nonclinical population: A test of the continuum hypothesis. Behaviour Research and Therapy, 43(8), 999-1010. https://doi.org/10.1016/j.brat.2004.06.017

Leonart, M. W., \& Radomsky, A. S. (2019). Responsibility causes reassurance seeking, too: An experimental investigation. Journal of Obsessive- 
Compulsive and Related Disorders, 20, 66-74.

https://doi.org/10.1016/j.jocrd.2017.10.005

Lyons, M., Aksayli, N. D., \& Brewer, G. (2018). Mental distress and language use: Linguistic analysis of discussion forum posts. Computers in Human Behavior, 87, 207-211. https://doi.org/10.1016/j.chb.2018.05.035

McKay, D., Abramowitz, J. S., Calamari, J. E., Kyrios M., Radomsky A., Sookman D., Taylor, S., \& Wilhelm, S. (2004). A critical evaluation of obsessive-compulsive disorder subtypes: Symptoms versus mechanisms. Clinical Psychology Review, 24(3), 283-313. https://doi.org/10.1016/j.cpr.2004.04.003

Melli, G., Bulli, F., Doron, G., \& Carreresi, C. (2018). Maladaptive beliefs in relationship obsessive compulsive disorder (ROCD): Replication and extension in a clinical sample. Journal of Obsessive-Compulsive and Related Disorders, 18, 47-53. https://doi.org/10.1016/j.jocrd.2018.06.005

Melli, G., Carraresi, C., Poli, A., Marazziti, D., \& Pinto, A. (2017). The role of guilt sensitivity in OCD symptom dimensions. Clinical Psychology 85 Psychotherapy, 24(5),1079-1089. https://doi.org/10.1002/cpp.2071

Miller, C. H., \& Hedges, D. W. (2008). Scrupulosity disorder: An overview and introductory analysis. Journal of Anxiety Disorders, 22(6), 1042-1058. https://doi.org/10.1016/j.janxdis.2007.11.004

Moulding, R., Aardema, F., \& O'Connor, K. P. (2014). Repugnant obsessions: A review of the phenomenology, theoretical models, and treatment of sexual and aggressive obsessional themes in OCD. Journal of ObsessiveCompulsive and Related Disorders, 3(2), 161-168. https://doi.org/10.1016/j.jocrd.2013.11.006

Olatunji, B. O., Sawchuk, C. N., Lohr, J. M., \& de Jong, P. J. (2004). Disgust domains in the prediction of contamination fear. Behavior Research and Therapy, 42(1), 93-104. https://doi.org/10.1016/S0005-7967(03)00102-5

Overbeek, T., Schruers, K., Vermetten, E., \& Griez, E. (2002). Comorbidity of obsessive-compulsive disorder and depression: Prevalence, symptom severity, and treatment effect. The Journal of Clinical Psychiatry, 63(12), 1106-1112. https://doi.org/10.4088/jcp.v63n1204

Pennebaker, J. W., Boyd, R. L., Jordan, K., \& Blackburn, K. (2015). The development and psychometric properties of LIWC2015. University of Texas at Austin.

Purdon, C. (2004). Cognitive-behavioral treatment of repugnant obsessions. Journal of Clinical Psychology, 60(11), 1169-1180. https://doi.org/10.1002/jclp.20081

Rude, S. S., Gortner, E. M., \& Pennebaker, J. W. (2004). Language use of depressed and depression-vulnerable college students. Cognition and Emotion, 18(8), 1121-1133. https://doi.org/10.1080/02699930441000030 
Schwartzman, C. M., Boisseau, C. L., Sibrava, N. J., Mancebo, M. C., Eisen, J. L., \& Rasmussen, S. A. (2017). Symptom subtype and quality of life in obsessive-compulsive disorder. Psychiatry Research, 249, 307-310. https://doi.org/10.1016/j.psychres.2017.01.025

Seo J. W., \& Kwon, S. M. (2013). Autogenous/reactive obsessions and their relationship with negative self-inferences. Journal of ObsessiveCompulsive and Related Disorders, 2(3), 316321. https://doi.org/10.1016/j.jocrd.2013.06.004

Shapiro, L. J., \& Stewart, E. S. (2011). Pathological guilt: A persistent yet overlooked treatment factor in obsessive-compulsive disorder. Annals of Clinical Psychiatry: Official Journal of the American Academy of Clinical Psychiatrists, 23(1), 63-70.

https://www.researchgate.net/publication/49833296_Pathological_Guilt _A_Persistent_Yet_Overlooked_Treatment_Factor_in_ObsessiveCompulsive_Disorder

Sheikhmoonesi, F., Hajheidari, Z., Masoudzadeh, A., Mohammadpour, R. A., \& Mozaffari, M. (2014). Prevalence and severity of obsessive-compulsive disorder and their relationships with dermatological diseases. Acta Medica Iranica, 52(7), 511-514.

Siev, J., Steketee, G., Fama, J. M., \& Wilhelm, S. (2011). Cognitive and clinical characteristics of sexual and religious obsessions. Journal of Cognitive Psychotherapy, 25(3), 167-176. https://doi.org/10.1891/0889$\underline{8391.25 .3 .167}$

Solem, S., Håland, A. T., Vogel, P. A., Hansen, B., \& Wells, A. (2009). Change in metacognitions predicts outcome in obsessive-compulsive disorder patients undergoing treatment with exposure and response prevention. Behaviour Research and Therapy, 47(4), 301-307. https://doi.org/10.1016/j.brat.2009.01.003

Tausczik, Y. R., \& Pennebaker, J. W. (2010). The psychological meaning of words: LIWC and computerized text analysis methods. Journal of Language and Social Psychology, 29(1), 24-54. https://doi.org/10.1177/0261927X09351676

Tolin, D. F., Abramowitz, J. S., Brigidi, B. D., \& Foa, E. B. (2003). Intolerance of uncertainty in obsessive-compulsive disorder. Journal of Anxiety Disorders, 17(2), 233-242. https://doi.org/10.1016/s0887-6185(02)00182-2

Vella-Zarb, R. A., Cohen, J. N., McCabe, R. E., \& Rowa, K. (2017). Differentiating sexual thoughts in obsessive-compulsive disorder from paraphilias and nonparaphilic sexual disorders. Cognitive and Behavioral Practice, 24(3), 342-352. https://doi.org/10.1016/j.cbpra.2016.06.007

Wheaton, M. G., Abramowitze, J. S., Berman, N. C., Riemann, B. C., \& Hale, L. R. (2010). The relationship between obsessive beliefs and symptom 
dimensions in obsessive-compulsive disorder. Behavior Research and Therapy, 48(10), 949-954. https://doi.org/10.1016/j.brat.2010.05.027

Williams, M. T., Chapman, L. K., Simms, J. V., \& Tellawi, G. (2017). Crosscultural phenomenology of obsessive-compulsive disorder. In J. S.

Abramowitz, D. McKay, \& E. A. Storch (Eds.), The Wiley handbook of obsessive compulsive disorders (pp. 56-74). Wiley Blackwell.

https://doi.org/10.1002/9781118890233.ch4

Yap, K., Mogan, C., \& Kyrios, M. (2012). Obsessive-compulsive disorder and comorbid depression: The role of OCD-related and non-specific factors. Journal of Anxiety Disorders, 26(5), 565-573.

https://doi.org/⒑1016/j.janxdis.2012.03.002 
Table 1.1

Keyness Results (RQs 1 \& 2)

\begin{tabular}{clccc}
\hline OCD Type & \multicolumn{1}{c}{ Rank Order } & $G^{2}$ & BIC & $\begin{array}{c}\text { BIC } \\
\text { Descriptor }\end{array}$ \\
\hline Autogenous & 1 sexual & $2,550.36$ & $2,535,57$ & Very strong \\
& 2 POCD & $2,359.52$ & $2,344.73$ & Very strong \\
& 3 harm & $1,497.00$ & $1,482.21$ & Very strong \\
& 4 HOCD & $1,425.18$ & $1,410.38$ & Very strong \\
& 5 gay & 989.94 & 975.15 & Very strong \\
6 attracted & 934.33 & 919.54 & Very strong \\
7 sex & 812.39 & 797.60 & Very strong \\
8 porn & 799.68 & 784.89 & Very strong \\
9 pedophile & 751.61 & 736.82 & Very strong \\
10 children & 727.73 & 712.94 & Very strong \\
& & & \\
1 checking & $3,818.78$ & $3,803.98$ & Very strong \\
2 contamination & $3,145.29$ & $3,130.50$ & Very strong \\
3 hands & $1,800.29$ & $1,785.50$ & Very strong \\
4 wash & $1,185.10$ & $1,170.30$ & Very strong \\
5 counting & $1,113.32$ & $1,098.53$ & Very strong \\
6 check & $1,058.47$ & $1,043.67$ & Very strong \\
7 clean & 961.29 & 946.50 & Very strong \\
8 contaminated & 902.85 & 888.06 & Very strong \\
9 washing & 888.94 & 874.15 & Very strong \\
10 door & 849.12 & 834.32 & Very strong \\
\hline
\end{tabular}


Figure 3.1

First-order Collocates of Sexual ( $R Q$ 3)

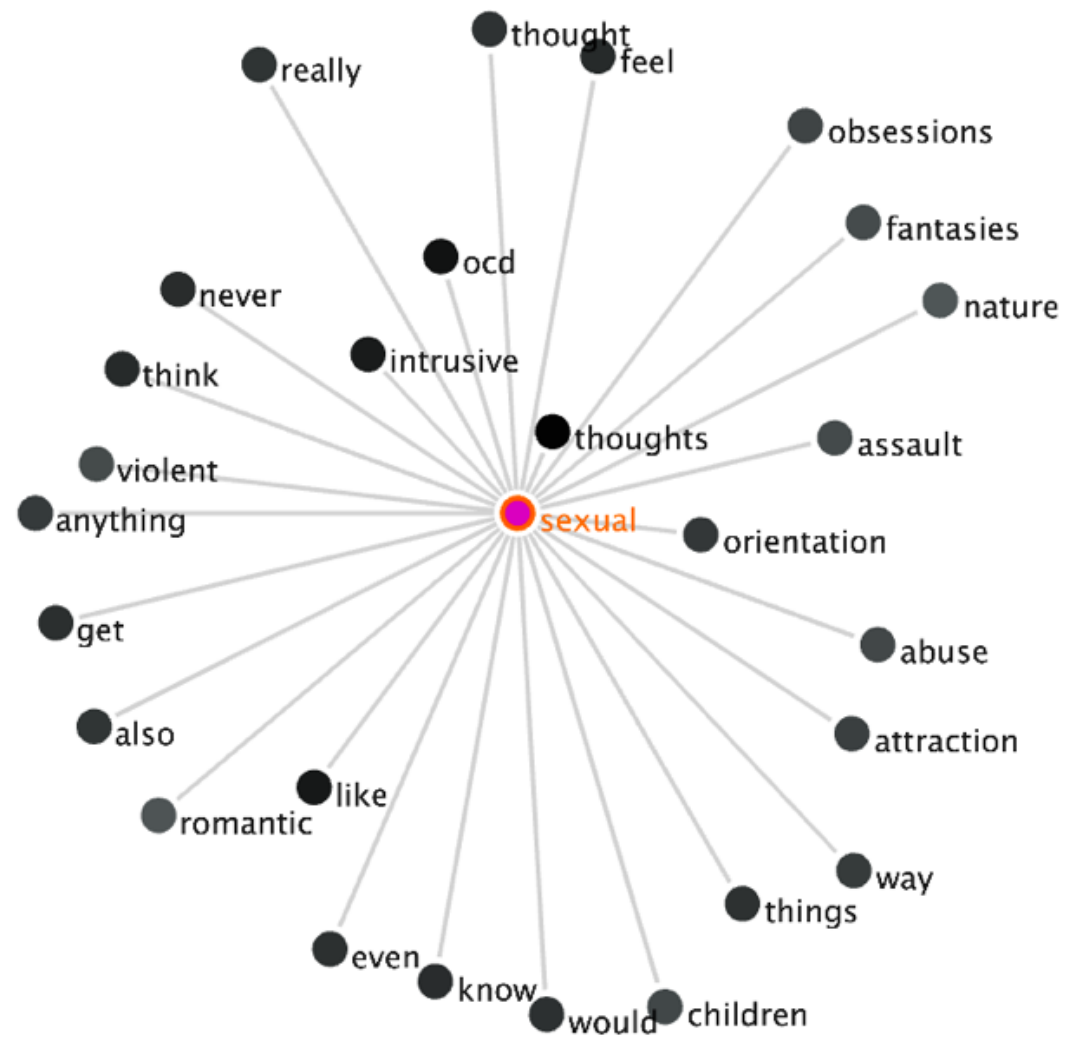


Figure 3.2

First-order Collocates of POCD (RQ 4)

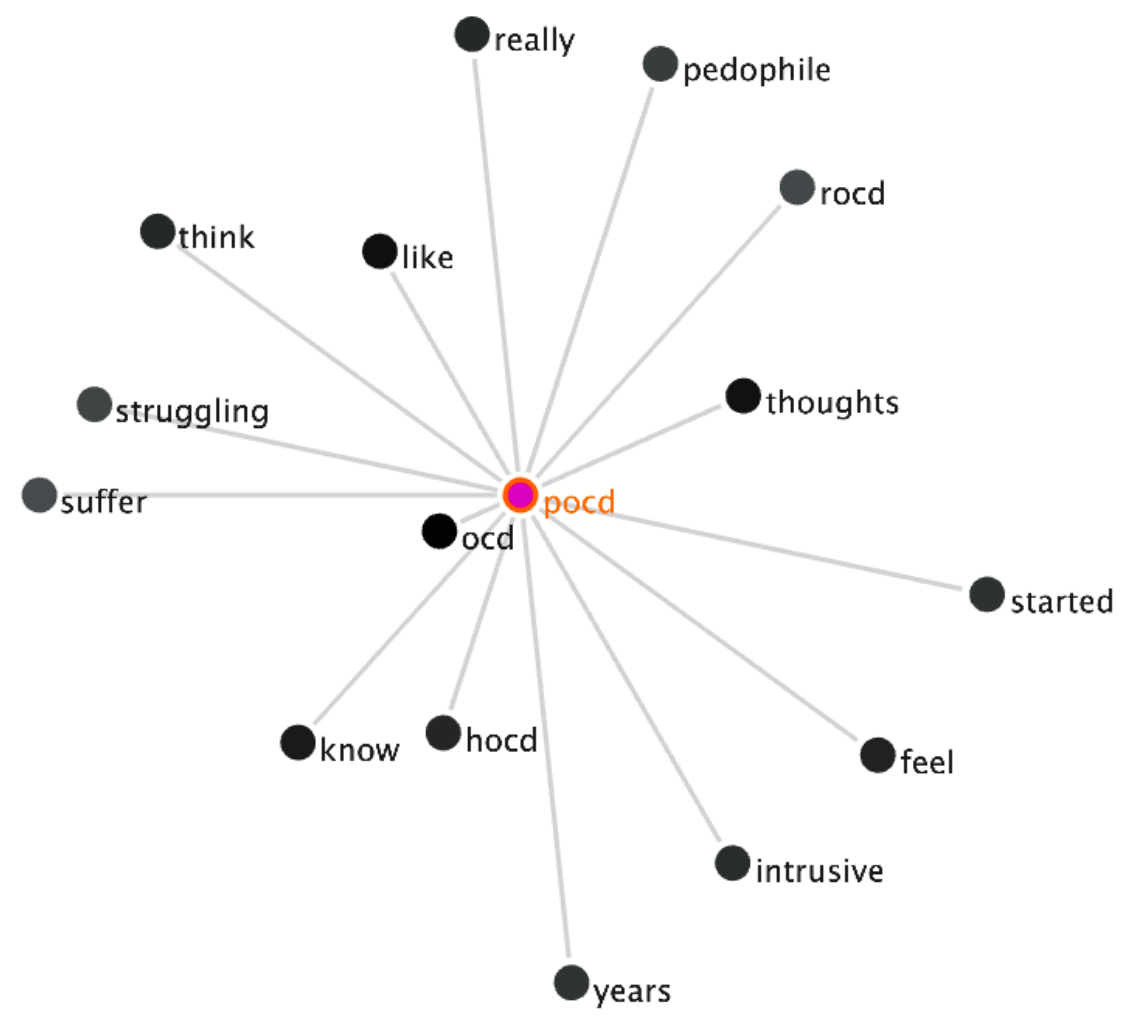


Figure 3.3

First-order Collocates of Checking (RQ 5)

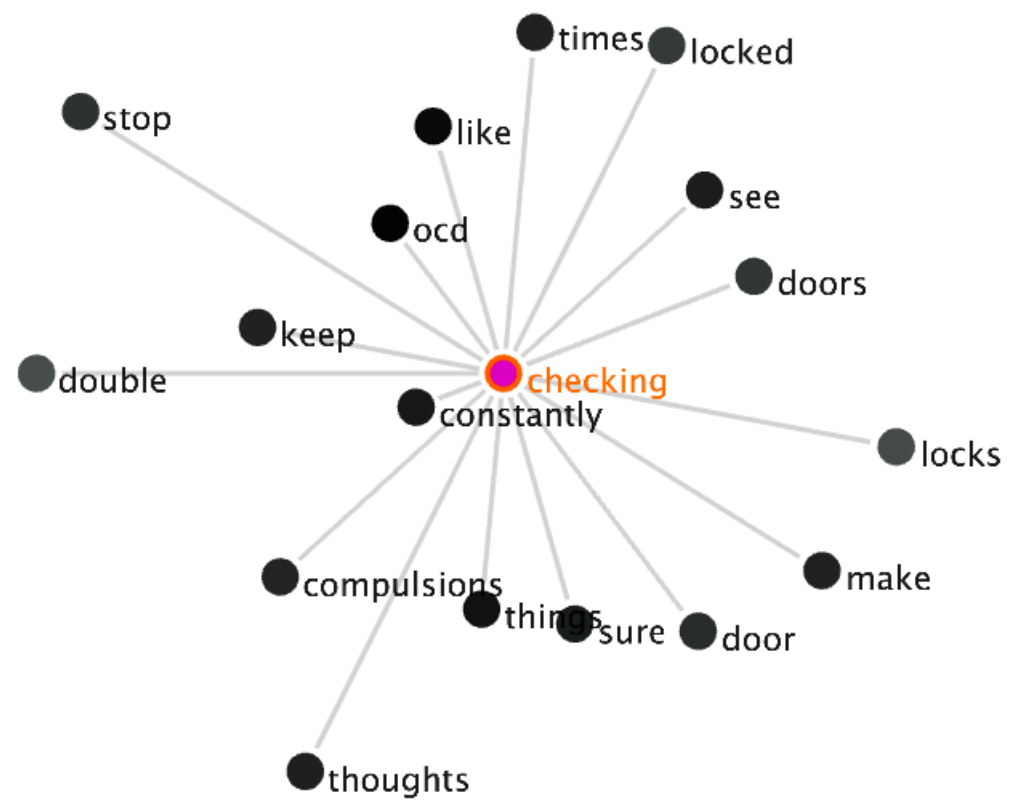


Figure 3.4

First-order Collocates of Contamination (RQ 6)

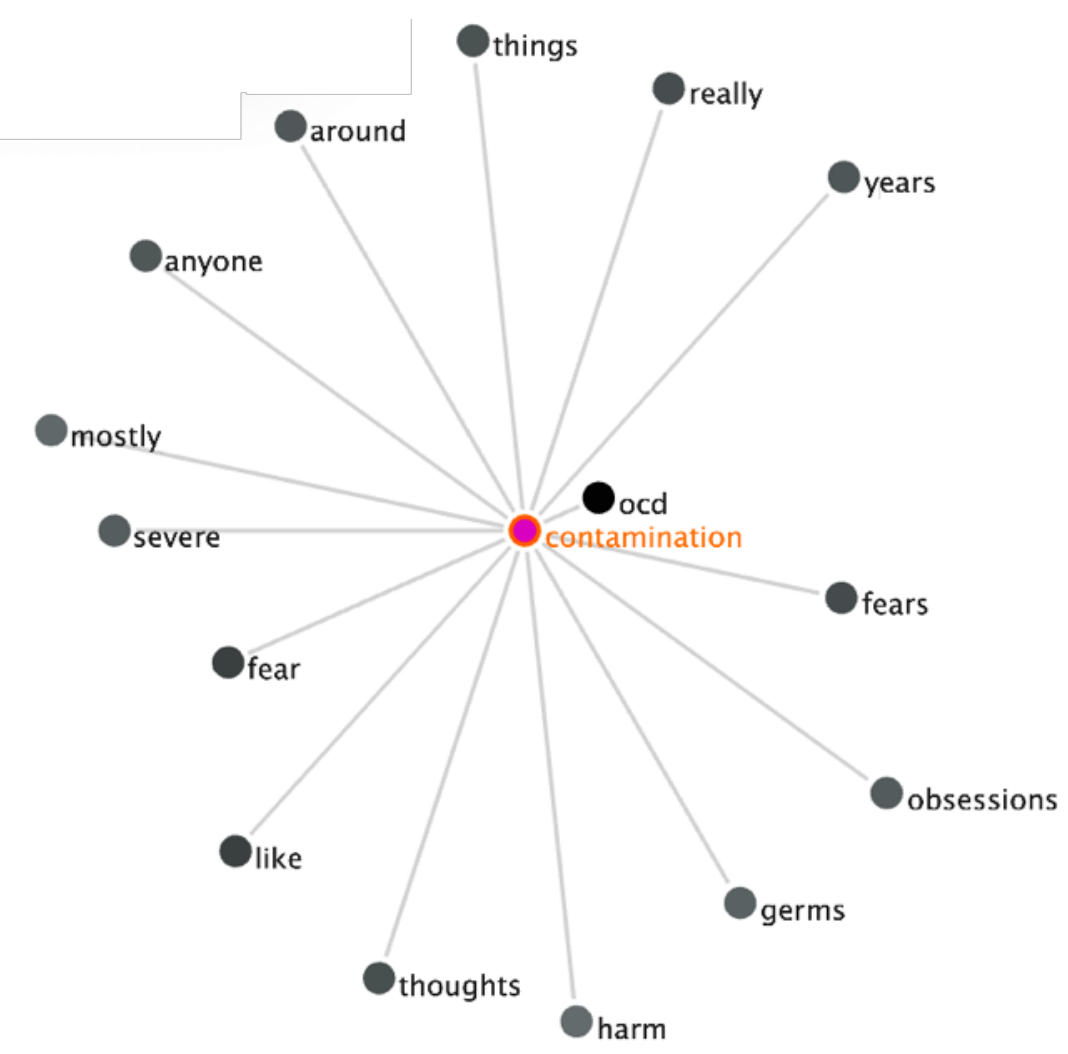

